\title{
Prácticas profesionales educativas con infancias en el marco de la pandemia de COVID-19: una experiencia de aprendizaje-servicio solidario
}

\author{
Macarena Abregú \\ Erica Molina \\ Universidad Abierta Interamericana, Argentina
}

\section{Resumen}

Se presenta una experiencia de aprendizaje-servicio en un proyecto de juegoteca virtual llamada LUDOTENA con niños/as de cinco a trece años en situación de vulnerabilidad social en el marco del aislamiento social, preventivo y obligatorio, debido a la pandemia de COVID-19 durante el periodo Junio-Diciembre de 2020. El marco institucional curricular en el cual se desarrolla es la Licenciatura de Terapia ocupacional de la Universidad Abierta Interamericana. El trabajo se lleva a cabo a partir de la construcción de redes interinstitucionales con referentes comunitarios que viven, conocen y construyen el desarrollo comunitario día a día. De esta manera, la relación con las familias se basa en una construcción de confianza con los referentes lo que promueve mayor afianzamiento a la propuesta. El proyecto tiene diversos propósitos. Por un lado, fortalecer la trayectoria académica de los estudiantes universitarios a partir de la participación en proyectos comunitarios reales, ajustados al contexto. Por el otro, promover la articulación de los marcos teóricos con la realidad social actual. Por último, se propone llevar a cabo acciones colectivas eficaces que contemplen las demandas socio-ocupacionales de las familias que forman parte del proyecto, así como también a las singularidades de los sujetos.

\section{Palabras clave}

Aprendizaje-servicio, terapia ocupacional, pandemia.

Fecha de recepción: 18/IX/2020

Fecha de aceptación: 6/XI/2020 


\title{
Professional practice placements with children within the context of the COVID-19 pandemic: a solidarity service- learning experience
}

\begin{abstract}
This paper discusses a service-learning experience conducted as part of a virtual game library project called LUDOTENA, which involved children aged between five and thirteen who were considered socially vulnerable in the context of social, preventive and compulsory isolation caused by the COVID-19 pandemic between June and December 2020. The project was conducted as part of the curriculum of the bachelor's degree in Occupational Therapy at the Inter-American Open University of Argentina. The project started with the creation of inter-institutional networks with community references who live, know and contribute to community development on a daily basis. Therefore, the relationship with the families is based on building trust with the community references mentioned, which contributed to successful implementation of the proposal. The project had three main purposes: firstly, to strengthen the academic performance of university students through their participation in authentic community projects that arise to meet needs created by social and health emergencies; secondly, to promote the articulation of theoretical frameworks within the current social reality and finally, to carry out effective collective actions that take into account the sociooccupational demands of the families that are part of the project, as well as the singularities of the subjects.
\end{abstract}

\section{Keywords}

Service-learning, occupational therapy, pandemic. 


\section{Introducción}

En Argentina el primer caso detectado de COVID-19 se informó el 3 de marzo en la Ciudad Autónoma de Buenos Aires (CABA). El 11 del mismo mes la Organización Mundial de la Salud (OMS) declara la pandemia por el brote de COVID-19.

A partir del 20 de marzo, el Estado Argentino decreta (Poder Ejecutivo Nacional, 2020) el aislamiento social, preventivo y obligatorio (ASPO) en todo el país para aquellas personas que no trabajan en sectores esenciales de la economía y la salud ${ }^{1}$ mediante el decreto 520/2020. El mismo sigue vigente a la fecha, en distintas fases, en todo el territorio argentino en función de la cantidad de contagios.

El 23 de junio de 2020, el Sistema de las Naciones Unidas en Argentina presentó el informe titulado "COVID-19 en Argentina: impacto socioeconómico y ambiental", en el cual se desarrolla, entre otros temas, el impacto de la pandemia sobre los derechos humanos (DDHH) de los grupos más vulnerables y los desafíos para niños y adolescentes.

En el mismo se menciona que:

En el segundo semestre de 2019, la pobreza afectaba al 53\% de los niños, y podría escalar al $58,6 \%$ hacia fines de 2020 . Los

\footnotetext{
${ }^{1}$ Los trabajadores que son esenciales pueden ir a sus lugares de trabajo y utilizar el transporte público. La lista de actividades se actualiza cada nueva etapa de aislamiento.
}

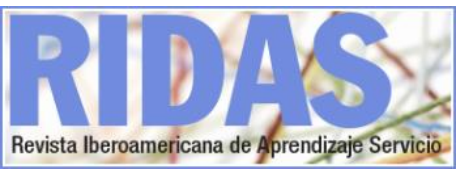

datos son proyecciones basadas en estimaciones de la caída del PIB y de la Encuesta Permanente de Hogares del Instituto Nacional de Estadística y Censos (INDEC). Por su parte, la pobreza extrema podría llegar al $16,3 \%$ hacia el final de 2020. En términos de volúmenes de población, implicaría que entre 2019 y 2020 la cantidad de niños y adolescentes pobres pasaría de 7 millones a 7,76 millones; la pobreza extrema, de 1,8 a 2,1 millones (Sistema de las Naciones Unidas en Argentina, 2020, p.25).

Este estudio señala que la mayor desigualdad e incidencia de la pobreza se ve en aquellos casos en los que los NNyA residen en hogares cuyo adulto responsable se encuentra desocupado, en un trabajo informal, con jefatura femenina $y / o$ son migrantes internacionales, aumentando aún más si residen en una villa o barrio popular.

El Registro Nacional de Barrios Populares de Argentina indica que 4,2 millones de personas viven en estos lugares: casi el $90 \%$ no cuenta con acceso formal al agua corriente, el $98 \%$ no tiene acceso a la red cloacal, el $64 \%$ a la red eléctrica formal y el $99 \%$ no accede a la red formal de gas natural (Sistema de las Naciones Unidas en Argentina, 2020, p.26).

La crisis de esta pandemia no sólo tendrá efectos a nivel sanitario, sino también ambientales, económicos y sociales. Y si bien la OMS señala como 
población de riesgo a las personas mayores o las personas con enfermedades preexistentes (pulmonares, cardíacas, diabetes, obesidad o que debiliten el sistema inmunitario), niños y adolescentes también se encuentran en situación de vulnerabilidad debido al COVID-19 viéndose afectados su crecimiento, desarrollo y bienestar biopsicosocial.

Al respecto, Luisa Brumana (Fondo de las Naciones Unidas para la Infancia [UNICEF], s.f.), representante de UNICEF Argentina afirma:

Los niños y las niñas son las víctimas ocultas del coronavirus. El COVID-19 no sólo puede enfermarlos: aunque no lo veamos, la pandemia también afecta su educación, los expone a la violencia e impacta en su salud mental y nutricional (párr.3).

El impacto del aislamiento puede afectar tanto su salud mental -que puede verse afectada por el estrés, depresión, pérdidas de familiares, temor al contagio- como la accesibilidad a los cuidados habituales (concurrir a una consulta médica o centros de salud), sumado a las dificultades en el acceso a alimentos y a la higiene necesaria -por falta de agua potable y saneamiento- señalado anteriormente, los expone tanto al COVID-19 como a otras enfermedades infectocontagiosas.

Cabe mencionar también la presión por tareas escolares que no pueden cumplir por falta de conexión, materiales o también por falta de apoyo. Muchos niños han asumido tareas del hogar dentro de sus responsabilidades diarias, significando menos tiempo de estudio u ocio lo que dificulta el seguimiento de la currícula y por tanto su reinserción al aula en un futuro.

A partir de la disposición del ASPO se suspendieron todas las actividades escolares y recreativas de manera presencial. Desde el Ministerio de Educación de la Nación Argentina se tomaron medidas para adaptar las currículas a las clases virtuales. Sin embargo, no poder asistir a las escuelas en muchos casos no implica solamente no acceder al aula sino también a otras actividades que allí se desarrollaban como comedores, merenderos, taller de juego, entre otras.

\section{Marco teórico}

\subsection{Marco institucional: Universidad y comunidad}

El proyecto LUDOTENA se realiza en el marco de la Carrera de Terapia Ocupacional (TO) de la Universidad Abierta Interamericana (UAI), en su sede de la Ciudad Autónoma de Buenos Aires.

El plan de estudio de dicha carrera está conformado por diversos espacios curriculares relacionados a problemáticas socio ocupacionales. LUDOTENA se lleva a cabo desde tres asignaturas: Terapia Ocupacional en Comunidad, Terapia Ocupacional en Prevención Primaria I y Práctica Profesional en Comunidad²; junto con la Secretaría de Extensión Universitaria.

2 Las tres asignaturas corresponden al segundo año de carrera. 
En los programas académicos, podemos encontrar el sentido y espíritu de las mencionadas asignaturas. Al respecto, Abregú (2020) expresa:

Dicho espacio curricular busca que el/la estudiante sea capaz de reflexionar críticamente sobre la realidad y problemáticas sociales que hoy convocan a la TO a nivel mundial. El análisis y reflexión teórica les permitirá pensar y llevar adelante prácticas transformadoras, desde la perspectiva crítica, es decir prácticas que fomenten el empoderamiento de los/as sujetos de derecho, que reconozca y respete la diversidad cultural, que no reproduzca estereotipos de género y que se posicione como un facilitador de la comunidad (p.2).

Según Tapia (2007):

Hablamos de aprendizaje-servicio cuando encontramos proyectos o programas, que tienen simultáneamente los dos tipos de objetivos, en donde está claro qué es lo que se aprende cuando se sale a la comunidad y está claro que se quiere brindar un servicio eficaz (p.18).

Desde la Juegoteca LUDOTENA, hemos construido objetivos en una doble dirección. Por un lado, entre los objetivos de aprendizaje para con los estudiantes voluntarios, podemos mencionar:

- Aprender a generar estrategias de intervención comunitaria de terapia ocupacional en el marco de una emergencia socio sanitaria.

- Implementar los contenidos aprehendidos en la cursada en la planificación del proyecto lúdico.

- Promover la participación en experiencias de extensión universitaria en el marco de su formación como terapistas ocupacionales.

Por otro lado, en cuanto a los objetivos de mejora comunitaria, proponemos:

- Que los niños en situación de apartheid ocupacional participen de un espacio de juego cuidado durante el aislamiento social.

- Promover aprendizajes significativos a través del juego.

- Promover autonomía para el desempeño de las áreas ocupacionales juego y educación.

Pensar y planificar los objetivos desde esta doble mirada, busca generar que el campo de lo teórico se pueda entrelazar con una práctica comunitaria transformadora real, y de esta manera construir un proceso educativo dialéctico.

En este sentido, Freire (1987) decía:

No debo admitirme, como profesional, "habitante" de un extraño mundo; mundo de técnicos y especialistas salvadores de los demás, dueños de la verdad, propietarios del saber que debe ser donado a los pandemia de COVID-19: una experiencia de aprendizaje-servicio solidario. RIDAS, Revista Iberoamericana de Aprendizaje Servicio, 10, 77-88. DOI10.1344/RIDAS2020.10.7 
"ignorantes e incapaces". Habitantes de un ghetto de donde salgo mecánicamente para salvar a los "perdidos" que están afuera. Si así procedo, no me comprometo verdaderamente, ni como profesional, ni como hombre. Simplemente me alieno (p.14).

\subsection{Marco normativo del proyecto}

LUDOTENA está fundamentado en las siguientes leyes nacionales:

- Ley 26.150²: Ley de Educación Sexual Integral (ESI) cuyo objetivo es garantizar la ESI de todos los niños y adolescentes.

- Ley 26.0614: Ley de protección Integral de los derechos de los niños y adolescentes, a través de la cual se establece que el Estado debe garantizar el cumplimiento de los derechos de los NNyA establecidos por el estado nacional como por tratados internacionales y reconoce a los NNyA como sujetos de derecho, instaurando el concepto de interés superior, cito: "la máxima satisfacción, integral y simultánea de los derechos y garantías reconocidos en esta ley".

${ }^{3}$ http://servicios.infoleg.gob.ar/infolegInternet/a nexos/120000-124999/121222/norma.htm ${ }^{4}$ http://www.jus.gob.ar/media/3108870/ley 260 61 proteccion de ni os.pdf

\section{RIDIS}

- Ley 26.2065: Ley de educación nacional, la cual establece como derecho personal y social enseñar y aprender, derecho que debe estar garantizado por el estado.

Partiendo de estas tres leyes como bases para la fundamentación, es importante mencionar los siguientes aspectos.

Según establece la Ley 26.061 todos Ios NNyA del territorio Nacional tienen derecho a la salud, a recibir una alimentación de calidad y al acceso a una vivienda digna.

Retomando lo mencionado anteriormente en relación con las condiciones de vida en los barrios populares y teniendo en cuenta que LUDOTENA se desarrolla en distintos barrios con esas características, nos preguntamos, ¿cómo hacer efectivo el ejercicio de este derecho?

En cuanto a la participación social, muchos niños en situación de apartheid ocupacional asistían a distintos dispositivos que ofrecían las organizaciones sociales del barrio, entre los que se encontraban espacios de juego, recreación y esparcimiento. La realidad actual, genera que esos espacios estén cerrados y para muchos niños implique la imposibilidad de jugar. Así mismo, la imposibilidad de ir a la escuela, no es reemplazada como en otros casos por clases virtuales ya que el acceso a la tecnología y la conectividad es en su mayoría baja o

${ }^{5}$ https://www.argentina.gob.ar/sites/default/files ley-de-educ-nac-58ac89392ea4c.pdf 
nula, y en otros casos estos niños no cuentan con el apoyo de un adulto para la realización de las tareas escolares o como mencionamos antes destinan este tiempo, que previo al aislamiento social, preventivo y obligatorio correspondía a la escolaridad, ahora a realizar las tareas del hogar. Se genera así una gran brecha de desigualdad que sin dudas se verá reflejada al momento de volver a la presencialidad.

La ley 26.150, nos acerca la posibilidad de acompañar a los NNyA en estos tiempos, a través de estrategias, por ejemplo, del cuidado del cuerpo y de las emociones. Distintas organizaciones como UNICEF y la Organización de las Naciones Unidas (ONU) han mencionado en diferentes artículos el aumento de la violencia intrafamiliar dirigida hacia los niños durante el período de aislamiento relacionándolo con el estrés y la ansiedad producidos por el encierro y la imposibilidad de acudir por ayuda. La violencia intrafamiliar es una forma de vulneración de los derechos humanos y de los derechos del niño, enmarcar este proyecto desde la ley de ESI nos permite generar estrategias de prevención con esos NNyA desde el juego en la virtualidad.

En cuanto a la ley 26.206, el Gobierno Nacional a través del Ministerio de Educación, el Consejo Federal de Educación y en coordinación con los organismos competentes de todas las jurisdicciones, estableció la suspensión del dictado de clases presenciales en los niveles inicial, primario y secundario en todas sus modalidades, e institutos de educación superior a partir del 16 de marzo a la actualidad.
A partir de ese momento, se tomaron algunas medidas de contención y acompañamiento a la escolaridad, entre ellas:

- Las instituciones educativas de gestión estatal entregan bolsones alimentarios cada quince días a aquellas familias que no pueden prescindir de la asistencia alimentaria.

- La ejecución del Programa "Seguimos educando" cuyo objetivo es colaborar con las condiciones para la continuidad de las actividades de enseñanza en el sistema educativo nacional a través de un sistema multiplataforma (Ministerio de Educación de Argentina, 2020).

- Se distribuyeron 7 millones de cuadernos impresos en todo el país. Los mismos ofrecen actividades acordes con los diferentes niveles de la escolaridad obligatoria -cuatro para nivel primario y dos para secundario- a los que se suman uno que comprende la edad de 0 a 3 años, y otro orientado para el uso en familia titulado Recreo.

Aun así, la complejidad del contexto socioeconómico y familiar genera que la continuidad de la escolaridad sea muy difícil. Por eso, podemos decir que las trayectorias escolares, entendidas como los "recorridos que realizan los sujetos en el sistema escolar, analizados en su relación con la expectativa que supone el diseño de tal sistema" (Terigi, 2017, s.p.), se han constituido en trayectorias 
no encauzadas. Según Terigi (2017) "itinerarios que no siguen el cauce diseñado por el sistema" (s.p.). En este caso, podríamos decir a causa de un factor pandémico.

\section{Descripción de la propuesta}

El proyecto LUDOTENA -jugar en cuarentena-, comenzó en el mes de junio de 2020, es decir tres meses después de declarado el aislamiento social preventivo y obligatorio (ASPO) y la suspensión de clases presenciales en todo el país. Se llevará a cabo hasta diciembre del corriente año.

Figura 1. Logo del proyecto

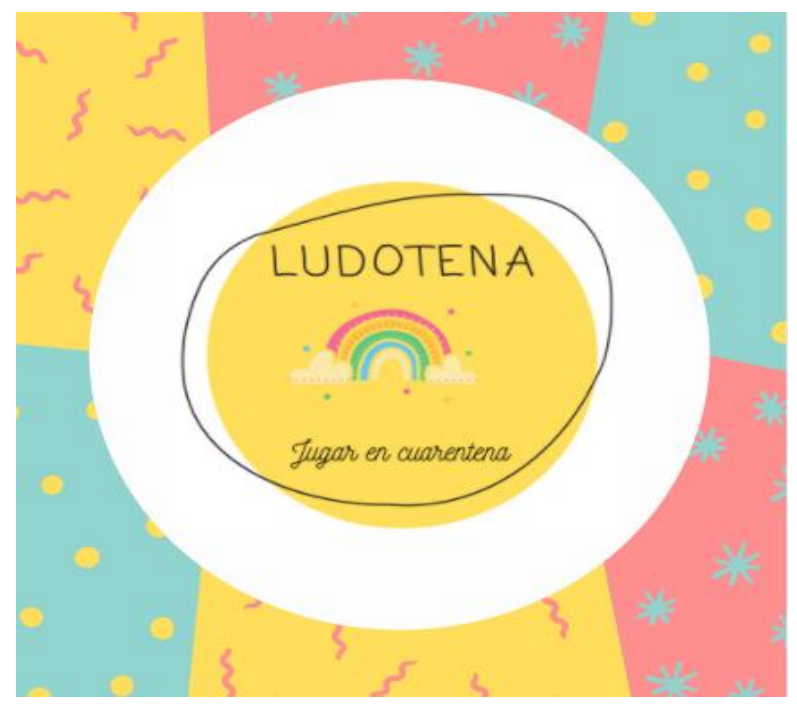

Fuente: elaboración propia

Está dirigido a niños de entre 5 y 13 años de edad, es decir quienes pertenecen a la etapa preescolar (nivel de educación inicial) y a primero y segundo ciclo de la educación de nivel primario.
Una de las características

fundamentales de estos niños es que viven en situación de vulnerabilidad socioeconómica y apartheid ocupacional ${ }^{6}$, entendido este último como:

[...] segregación de grupos de personas mediante la restricción o negación de su acceso a una participación digna y significativa en las ocupaciones de la vida diaria, basada en la raza, color, discapacidad, procedencia nacional, edad, sexo, orientación sexual, religión, creencias políticas, estatus en la sociedad u otras características [...] (Kronenberg, Simó Algado, y Pollard, 2006, p.66).

La juegoteca virtual consiste en la realización de dos encuentros semanales a través de videollamadas o el uso de plataformas como zoom, en el cual comparten junto a los voluntarios un momento de juego. Cada llamada es realizada a una familia, aunque en algunas ocasiones se han realizado encuentros compartidos entre varias. Los costos materiales para la conectividad en el momento de la videollamada se encuentran a cargo del proyecto.

\footnotetext{
${ }^{6}$ Actualmente los niños que participan en los proyectos residen en el Barrio 31, Villa 1-11-14, Villa 15, Villa 20 de la Ciudad Autónoma de Buenos Aires, y en el Partido de General Rodríguez de la Provincia de Buenos Aires.
} pandemia de COVID-19: una experiencia de aprendizaje-servicio solidario. RIDAS, Revista Iberoamericana de Aprendizaje Servicio, 10, 77-88. DOI10.1344/RIDAS2020.10.7 
Ilustración 1. Imágenes de las videollamadas realizadas en el marco del proyecto

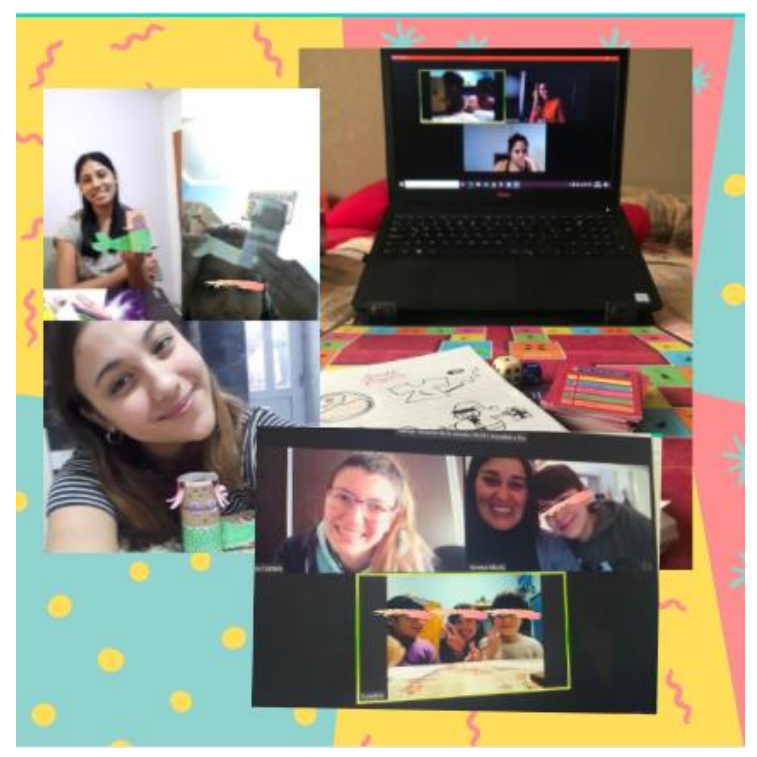

Fuente: elaboración propia

Actualmente, se encuentran participando 30 familias y 37 niños. Por otro lado, son 26 estudiantes, en su mayoría cursando en la actualidad las materias mencionadas, una graduada y 4 estudiantes de años posteriores.

Durante el mes de agosto, se ha realizado una convocatoria abierta para voluntarios con perfil de experiencia en disciplinas vinculadas a la educación y/o salud. Hasta el momento, se han inscripto 29, que comenzarán a realizar encuentros de juego a partir de octubre 2020 con familias que se encuentran en espera de una vacante. En relación con los estudiantes voluntarios cursantes deben cumplir con los criterios de evaluación del espacio curricular de práctica profesional en comunidad: análisis institucionales $\mathrm{y} / \mathrm{u}$ organizacionales, diseño de proyectos comunitarios para cada familia jugadora, diseñar criterios de evaluación del proyecto.

Para la convocatoria de familias, se han gestionado redes interinstitucionales con distintas organizaciones sociales y/o equipos territoriales del Estado, quienes convocaron y seleccionaron a las familias participantes. Entre las organizaciones con las que articulamos podemos mencionar:

- Servicio Social del Hospital Nacional Sommer: proyecto R.A.Y.U.E.L.A.S (Respuestas alternativas y unificación de estrategias legitimables para actores sociales), ubicado en el Partido General Rodríguez, Provincia de Buenos Aires.

- Unidad Técnica de abordaje integral de la Subsecretaría de Promoción de derechos para la niñez, la adolescencia y la familia. Perteneciente al Ministerio Nacional de Desarrollo Social.

- Casa de la Mujer de la Villa 111 14, CABA.

- Comedor Comunitario "Los Solcitos", Lugano, CABA.

En relación con la formación teórica de los estudiantes voluntarios, en su mayoría se encuentra cursando las asignaturas correspondientes o lo han realizado en años anteriores. Decidimos fortalecer su rol a través de las siguientes acciones:

- Conversatorios con referentes comunitarios y/o funcionarios del Estado formados en materia de pandemia de COVID-19: una experiencia de aprendizaje-servicio solidario. RIDAS, Revista Iberoamericana de Aprendizaje Servicio, 10, 77-88. DOI10.1344/RIDAS2020.10.7 
infancias y derechos.

- Talleres participativos para el uso de plataformas y recursos tecnológicos para el armado de juegos.

- Clases especiales con especialistas en aprendizaje, neurociencias, educación sexual integral y juegotecas.

- Confección y distribución de tres cuadernillos de recopilación bibliográfica en torno a tres ejes: juegotecas, educación sexual integral e infancias desde la perspectiva de derechos.

Por último, con relación a la gestión y coordinación del proyecto en general, podemos destacar cuatro etapas principales, las cuales se reflejan en la figura 2:

- Etapa preliminar: armado y gestión administrativa, comprendida por convocatoria y reuniones con organización, voluntarios y familias. Puesta en común de intereses y objetivos en relación con el proyecto.

\section{- Etapa Inicial: una vez} confirmados los participantes se realizó la adjudicación de las familias y el armado de equipos de trabajo para facilitar la dinámica de este.

- Implementación: la puesta en marcha del proyecto. Comienzan los encuentros virtuales.

Fortalecimiento: luego de un período de implementación se realiza una reevaluación y observación de los resultados a partir del cual se decide realizar una nueva convocatoria a voluntarios externos, ampliando así el alcance del proyecto. Paralelamente se comienza con los encuentros de formación para los voluntarios.

Figura 2. Etapas del proyecto

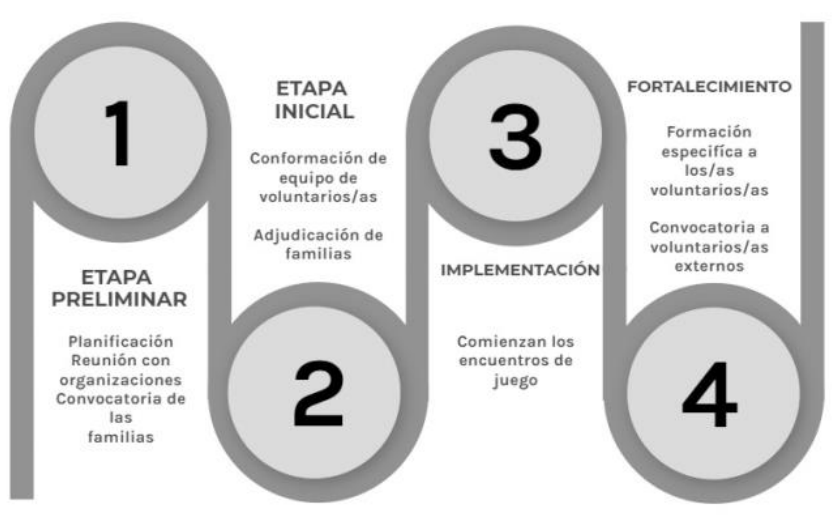

Fuente: elaboración propia

Entre los desafíos que nos encontramos en este proceso podemos mencionar, por un lado, relacionados a las problemáticas estructurales de la comunidad como las dificultades en el acceso a internet, la baja calidad de conexión y cortes de suministro eléctrico. Por otro lado, desafíos directamente vinculados con la puesta en práctica de los recursos didácticos con los que cuentan los voluntarios. Estas situaciones las están fortaleciendo a través del acompañamiento continuo para la planificación de encuentros compartiendo herramientas apropiadas.

Además, en el caso del Hospital Nacional Sommer, conseguimos que el mismo habilitará un router específico para las familias que participan del proyecto, algo que modificó la calidad de las videollamadas automáticamente. 


\section{Conclusiones}

A lo largo de este artículo hemos expuesto no sólo las condiciones actuales que atraviesan niños y adolescentes en nuestro país debido a la realidad socioeconómica sino también a cómo se han enfatizado $\mathrm{y} / \mathrm{o}$ profundizado aún más en este contexto de pandemia y aislamiento social en el que nos encontramos.

Hemos explicado cómo a partir de estas problemáticas encontramos ampliamente vulnerados sus derechos, siendo este uno de los disparadores para la creación del proyecto LUDOTENA, y cómo a partir de esto encontramos una oportunidad en medio de la crisis para generar un nuevo espacio de aprendizaje para aquellos alumnos y graduados que participan como voluntarios/as.

LUDOTENA busca ser un espacio de encuentro con niños y adolescentes donde a través del juego podamos acompañar sus trayectorias educativas y promover sus derechos. Pero también será un espacio de encuentro para los voluntarios en diferentes maneras: un lugar para encontrarse en el juego desde su adultez, para repensar sus prácticas profesionales y estudiantiles, para aprender en el intercambio con un otro, para crear nuevas formas de intervención en un contexto de aislamiento donde las relaciones se crean y sostienen a través de una pantalla.

La pandemia COVID-19 dejará muchas consecuencias para nuestra sociedad. Creemos que las universidades no quedan por fuera de los actores sociales que tendrán que colaborar en la reconstrucción del tejido social. Los proyectos de aprendizaje servicio se convierten en una oportunidad para esta singular, compleja y hermosa tarea.

\section{Referencias bibliográficas}

Abregú, M. (2020). Programa de la Cátedra Prevención Primaria. Plan de estudio P2-17. Buenos Aires, Argentina: Universidad Abierta Interamericana.

Fondo de las Naciones Unidas para la Infancia [UNICEF]. (s.f.). UNICEF responde a la pandemia del COVID-19 en Argentina. Recuperado de: https://www.unicef.org/argentina/unice f-responde-la-pandemia-del-covid-19en-argentina

Freire, P. (1987). Educación y cambio. Buenos Aires, Argentina: Búsqueda.

Kronenberg, F., Simó Algado, S. y Pollard, N. (2006). Terapia ocupacional sin fronteras: aprendiendo del espíritu de supervivientes. Madrid, España: Panamericana.

Ministerio de Educación de Argentina (2020). Resolución 106 de 2020 por la cual se crea el Programa "Seguimos educando" en el ámbito del Ministerio de Educación. Ciudad de Buenos Aires, Argentina: Ministerio de Educación. Recuperado de: https://www.boletinoficial.gob.ar/detall eAviso/primera/226751/20200316

Poder Ejecutivo Nacional (2020). Decreto 520 de 2020 que establece la medida de "distanciamiento social, preventivo y obligatorio" en los pandemia de COVID-19: una experiencia de aprendizaje-servicio solidario. RIDAS, Revista Iberoamericana de Aprendizaje Servicio, 10, 77-88. DOI10.1344/RIDAS2020.10.7 
términos ordenados por el presente decreto, para todas las personas que residan o transiten en los aglomerados urbanos $y$ en los partidos $y$ departamentos de las provincias argentinas en tanto estos verifiquen en forma positiva determinados parámetros epidemiológicos y sanitarios. Ciudad de Buenos Aires:

Poder Ejecutivo Nacional. Recuperado de:

https://www.boletinoficial.gob.ar/detall eAviso/primera/230245/20200608

Sistema de Naciones Unidas en Argentina. (2020). COVID-19 en Argentina: impacto socioeconómico y ambiental. Recuperado de:

https://www.onu.org.ar/stuff/InformeCOVID-19-Argentina.pdf

Tapia M. N. (2007). El AprendizajeServicio en las OSC. En A. González y L. Walther (Comps.), Aprendizaje y servicio solidario en las organizaciones de la sociedad civil; Actas de la Primera Jornada Abierta para OSC del campo educativo. Buenos Aires, Argentina. Recuperado de:

http://roserbatlle.net/wpcontent/uploads/2009/06/2007 actas osc i1.pdf

Terigi, F. (2017). Trayectorias educativas y trayectorias escolares. Especialización docente en Políticas Socioeducativas. Módulo EPS, Clase 5. Recuperado de:

https://docplayer.es/128997715-

Especializacion-docente-en-politicassocioeducativas-modulo-eps-clase-

$\underline{\text { 5.html }}$ 